\title{
Wichtige sicherheitsrelevante Information zu XELJANZ ${ }^{\circledR}$ (Tofacitinib)
}

\section{Erste Ergebnisse einer klinischen Studie deuten auf ein erhöhtes Risiko für schwerwiegende unerwünschte kardiovaskuläre Ereignisse und maligne Erkrankungen (ohne NMSC) bei der Verwendung von Tofacitinib im Vergleich zu TNF-alpha-Inhibitoren hin.}

Sehr geehrte Damen und Herren,

In Absprache mit Swissmedic möchte Pfizer Sie über Folgendes informieren:

\section{Zusammenfassung}

- Vorläufige Daten aus einer abgeschlossenen klinischen Studie bei Patienten mit rheumatoider Arthritis (A3921133) deuten auf ein höheres Risiko für schwerwiegende unerwünschte kardiovaskuläre Ereignisse (MACE) und maligne Erkrankungen (mit Ausnahme von nicht melanozytärem Hautkrebs [NMSC]) unter der Behandlung mit Tofacitinib im Vergleich zu Patienten, die mit einem TNF-alpha-Inhibitor behandelt wurden.

- Bei der Entscheidung, ob Sie Patienten Tofacitinib verschreiben oder ob diese weiterhin die Therapie fortsetzen, sollten Sie auch künftig den Nutzen und die Risiken einer Anwendung von Tofacitinib abwägen. Beachten Sie weiterhin die Empfehlungen in der Arzneimittelinformation von Tofacitinib.

- Informieren Sie die Patienten, dass sie die Einnahme vonTofacitinib nicht ohne vorherige Rücksprache mit ihrem Arzt beenden sollten und dass sie sich bei Fragen oder Bedenken an ihren Arzt wenden sollen.

- Die weitere Auswertung der Daten aus der Studie A3921133 und deren möglichen Relevanz für die Arzneimittelinformation vonTofacitinib wird derzeit von Swissmedic und anderen Aufsichtsbehörden vorgenommen. Die endgültigen Schlussfolgerungen und Empfehlungen werden nach Abschluss der Bewertung bekanntgegeben.

Ähnliche Mitteilungen wurden am 4.2.2021 von der US FDA und am 24.3.2021 von der EMA an verschreibende Ärzte in den USA beziehungsweise in der EU verschickt.

\section{Hintergrundinformationen}

Tofacitinib ist ein JAK-Inhibitor, der indiziert ist für die Behandlung

- der mittelschweren bis schweren aktiven rheumatoiden Arthritis (RA) oder aktiven Psoriasis-Arthritis (PsA) bei erwachsenen Patienten, die auf ein oder mehrere krankheitsmodifizierende Antirheumatika unzureichend angesprochen oder diese/s nicht vertragen haben;
- erwachsener Patienten mit mittelschwerer bis schwerer aktiver Colitis ulcerosa (CU), die auf eine konventionelle Therapie oder einen TNF-alpha-Inhibitor (TNFi) unzureichend angesprochen haben, nicht mehr darauf ansprechen oder diese nicht vertragen haben.

Langzeitstudie A3921133 zur Überwachung der Sicherheit bei Patienten mit rheumatoider Arthritis

Die Studie ORAL Surveillance (A3921133) ist eine grosse $(\mathrm{N}=4362)$ randomisierte, aktiv kontrollierte klinische Prüfung zur Bewertung der Sicherheit (PASS-Studie) von Tofaciti- nib in zwei Dosierungen (5 mg zweimal täglich und $10 \mathrm{mg}$ zweimal täglich) im Vergleich zu einem TNFi bei Patienten mit RA, die 50 Jahre oder älter waren und mindestens einen weiteren kardiovaskulären Risikofaktor aufwiesen (im Prüfplan definiert als aktuelle/r Raucher/in, arterielle Hypertonie, HighDensity-Lipoprotein [HDL] $<40$ mg/dl, Diabetes mellitus, vorbestehende koronare arterielle Verschlusskrankheit, familiäre Vorbelastung für vorzeitige koronare Herzerkrankung, extraartikuläre Manifestationen der RA), von denen einige auch bekannte Risikofaktoren für eine Krebserkrankung sind.

Die primären Endpunkte der Studie bestanden aus adjudizierten MACE und adjudizierten malignen Erkrankungen (ausser NMSC). Die Studie ist eine ereignisgesteuerte Studie, die auch eine Nachbeobachtung von mindestens 1500 Patienten über 3 Jahre erfordert. Vordefinierte Nichtunterlegenheitskriterien wurden für diese primären Endpunkte nicht erfüllt, und die Studie konnte nicht zeigen, dass Tofacitinib den TNFi nicht unterlegen («nicht schlechter») ist. Die Ergebnisse deuten darauf hin, dass diese Risiken mit beiden zugelassenen Dosierungen ( $5 \mathrm{mg}$ zweimal täglich und $10 \mathrm{mg}$ zweimal täglich, letztere nur bei Colitis ulcerosa zugelassen) bestehen.

In der Primäranalyse wurden die Daten von 135 Patienten mit adjudizierten schwerwiegenden unerwünschten kardiovaskulären Ereignissen (MACE) und 164 Patienten mit adjudizierten Krebserkrankungen (ausser NMSC) ausgewertet. Die am häufigsten gemeldeten MACE waren Myokardinfarkte. Die am häufigsten gemeldete Krebserkrankung (ausser NMSC) war Lungenkrebs. Bei Patienten mit einer höheren Prävalenz von bekannten Risikofaktoren für MACE und Krebserkrankungen (z.B. höheres Alter, Rauchen) wurden in allen 
Behandlungsgruppen mehr Ereignisse beobachtet.

Derzeit erfolgt eine weiterführende Auswertung der Daten aus der Studie A3921133 und deren möglichen Auswirkungen auf die Arzneimittelinformation von Tofacitinib durch
Swissmedic und andere Aufsichtsbehörden. Die endgültigen Schlussfolgerungen und Empfehlungen werden kommuniziert, sobald diese Auswertung abgeschlossen ist. Die aktualisierte Arzneimittelinformation wird unter www.swissmedicinfo.ch publiziert.
Adjudizierte MACE*

\begin{tabular}{|c|c|c|c|c|}
\hline & $\begin{array}{r}2 \times \text { täglich } 5 \mathrm{mg} \\
\text { Tofacitinib }\end{array}$ & $\begin{array}{r}2 \times \text { täglich } 10 \mathrm{mg} \\
\text { Tofacitinib** }\end{array}$ & $\begin{array}{r}\text { Beide Tofacitinib- } \\
\text { Dosen } \\
\text { zusammen }\end{array}$ & $\begin{array}{l}\text { TNF-alpha- } \\
\text { Inhibitoren }\end{array}$ \\
\hline Patienten insgesamt & 1455 & 1456 & 2911 & 1451 \\
\hline $\begin{array}{l}\text { Anzahl Patienten mit erstem } \\
\text { Ereignis innerhalb des Risiko- } \\
\text { zeitraums*** }(\%)\end{array}$ & $47(3,23)$ & $51(3,50)$ & $98(3,37)$ & $37(2,55)$ \\
\hline Patientenjahre & 5166,32 & 4871,96 & 10038,28 & 5045,27 \\
\hline $\begin{array}{l}\text { IR (95\% KI) } \\
\text { (Anzahl Patienten mit Ereignis- } \\
\text { sen/100 Patientenjahre) }\end{array}$ & $0,91(0,67 ; 1,21)$ & $1,05(0,78 ; 1,38)$ & $0,98(0,79 ; 1,19)$ & $0,73(0,52 ; 1,01)$ \\
\hline $\begin{array}{l}\text { HR (95\% KI) für Tofacitinib } \\
\text { vs. TNF-alpha-Inhibitoren }\end{array}$ & $1,24(0,81 ; 1,91)$ & $1,43(0,94 ; 2,18)$ & $\begin{array}{r}1,33(0,91 \\
1,94)^{* * * *}\end{array}$ & \\
\hline
\end{tabular}

(*) Basierend auf Cox' proportionalem Hazard-Modell.

${ }^{* *}$ Die Behandlungsgruppe mit $2 \times$ täglich $10 \mathrm{mg}$ Tofacitinib umfasst Patienten, die im Rahmen einer Studienänderung im Februar 2019 von $2 \times$ täglich $10 \mathrm{mg}$ auf $2 \times$ täglich $5 \mathrm{mg}$ umgestellt wurden.

$\left.{ }^{* * *}\right)$ Der Risikozeitraum erstreckte sich vom Beginn der Therapie bis 60 Tage nach der letzten Dosis.

(****) Für den primären Vergleich der kombinierten Tofacitinib-Dosen mit TNF-alpha-Inhibitoren wurde das Nichtunterlegenheitskriterium nicht erreicht, da die Obergrenze des $95 \% \mathrm{KI}$ oberhalb der vordefinierten Nichtunterlegenheitsgrenze von 1,8 lag, das heisst: 1,94 >1,8.

$\mathrm{HR}=$ Hazard Ratio, IR = Inzidenzrate, $\mathrm{KI}=$ Konfidenzintervall, TNF = Tumornekrosefaktor.

Adjudizierte Malignitäten ausser NMSC*

\begin{tabular}{|c|c|c|c|c|}
\hline & $\begin{array}{r}2 \times \text { täglich } 5 \mathrm{mg} \\
\text { Tofacitinib }\end{array}$ & $\begin{array}{l}2 \times \text { täglich } 10 \mathrm{mg} \\
\text { Tofacitinib** }\end{array}$ & $\begin{array}{r}\text { Beide Tofacitinib- } \\
\text { Dosen } \\
\text { zusammen }\end{array}$ & $\begin{array}{l}\text { TNF-alpha- } \\
\text { Inhibitoren }\end{array}$ \\
\hline Patienten insgesamt & 1455 & 1456 & 2911 & 1451 \\
\hline $\begin{array}{l}\text { Anzahl Patienten mit erstem } \\
\text { Ereignis innerhalb des Risiko- } \\
\text { zeitraums*** }(\%)\end{array}$ & $62(4,26)$ & $60(4,12)$ & $122(4,19)$ & $42(2,89)$ \\
\hline Patientenjahre & 5491,48 & 5311,71 & 10803,19 & 5482,30 \\
\hline $\begin{array}{l}\text { IR (95\% KI) } \\
\text { (AnzahI Patienten mit Ereignis- } \\
\text { sen/100 Patientenjahre) }\end{array}$ & $1,13(0,87 ; 1,45)$ & $1,13(0,86 ; 1,45)$ & $1,13(0,94 ; 1,35)$ & $0,77(0,55 ; 1,04)$ \\
\hline $\begin{array}{l}\text { HR (95\% KI) für Tofacitinib } \\
\text { vs. TNF-alpha-Inhibitoren }\end{array}$ & $1,47(1,00 ; 2,18)$ & $1,48(1,00 ; 2,19)$ & $\begin{array}{l}1,48(1,04 \\
2,09)^{* * * *}\end{array}$ & \\
\hline
\end{tabular}

(*) Basierend auf Cox' proportionalem Hazard-Modell.

(**) Die Behandlungsgruppe mit $2 \times$ täglich $10 \mathrm{mg}$ Tofacitinib umfasst Patienten, die im Rahmen einer Studienänderung im Februar 2019 von $2 \times$ täglich $10 \mathrm{mg}$ auf $2 \times$ täglich 5 mg umgestellt wurden.

$\left.{ }^{(* * *}\right)$ Der Risikozeitraum umfasste das gesamte verfügbare Follow-up, unabhängig von der Exposition gegenüber dem Wirkstoff.

(****) Für den primären Vergleich der kombinierten Tofacitinib-Dosen mit TNF-alpha-Inhibitoren wurde das Nichtunterlegenheitskriterium nicht erreicht, da die Obergrenze des $95 \% \mathrm{KI}$ oberhalb der vordefinierten Nichtunterlegenheitsgrenze von $1,8 \mathrm{lag}$, das heisst: $2,09>1,8$.

$\mathrm{HR}=$ Hazard Ratio, IR = Inzidenzrate, $\mathrm{KI}=$ Konfidenzintervall, TNF = Tumornekrosefaktor.

\section{Empfehlungen für Fachpersonen}

Bei der Entscheidung über eine Verordnung des Arzneimittels oder eine Fortsetzung der Behandlung sollten verschreibende Ärzte den Nutzen gegenüber den Risiken von Tofacitinib abwägen und Patienten über die Risiken und den Nutzen von Tofacitinib beraten. Empfehlen Sie Ihren Patienten, die Einnahme von Tofacitinib nicht ohne vorherige Rücksprache mit dem Arzt zu beenden und sich bei Fragen oder Bedenken an den Arzt zu wenden. Verschreibende Ärzte sollten weiterhin die Empfehlungen in der Schweizer Fachinformation von Tofacitinib beachten.

\section{Meldung unerwünschter Arzneimittel- wirkungen}

Für Meldungen über unerwünschte Arzneimittelwirkungen (UAW) empfiehlt Swissmedic, das dafür entwickelte Meldeportal Electronic Vigilance System (ElViS) zu verwenden. Alle erforderlichen Informationen hierzu sind unter www.swissmedic.ch zu finden.

\section{Kontaktangaben}

Sollten Sie weitere Fragen haben oder zusätzliche Informationen benötigen, so wenden Sie sich bitte an Pfizer Medical Information, Tel.+41434957111 oder medical.information@ pfizer.com

\section{Beilagen}

Für weitere Informationen verweisen wir auch auf die Arzneimittelfachinformation von $\mathrm{XELJANZ}^{\circledR}$ unter

http://www.swissmedicinfo.ch

\section{Mit freundlichen Grüssen}

Dr. med. Rahel Troxler Saxer Medical Director

Dominique Haiden Deputy Head Regulatory Affairs

Pfizer AG

Schärenmoosstrasse 99

8052 Zürich 